\title{
Risk Analysis with Information Described in Natural Language
}

\author{
Chongfu Huang ${ }^{1,2}$ \\ ${ }^{1}$ Key Laboratory of Environmental Change and Natural Disaster \\ The Ministry of Education of China, Beijing Normal University \\ No.19 Xinjiekouwai Street Beijing 100875, China \\ ${ }^{2}$ Research Center for System Simulation and Disaster Modeling \\ Academy of Disaster Reduction and Emergency Management \\ Ministry of Civil Affairs \& Ministry of Education \\ hchongfu@bnu. edu.cn
}

\begin{abstract}
In this paper, we give a new definition of risk: a scene in the future associated with some adverse incident. The simplest scene is that, a stone is hooked over our head. When a wind comes, the stone would fall down on our head. In many cases, what can we do is to approximately represent risks. In this paper, we suggest an approach to infer risks with information described in natural language. With fuzzy logic, we give a sample to verify that the suggested approach is more flexible and effective.
\end{abstract}

Keywords: Risk Analysis; Natural Language; Fuzzy Logic.

\section{Introduction}

All goals of developing science and technology are specified three categorizations for daily life: (1) Producing high quality goods with low variable costs; (2) Exploring the mystery of the world; (3)Providing safety.

Five years ago, the world witnessed an unprecedented attack on the civilized world. The attacks of 9/11 shocked men and women everywhere, and images from that day remain etched in the minds of all of us who saw them. On that dreadful day, al-Qaida's hijack crews stole the future from nearly 3000 innocent people, devastating the lives of their families and friends.

Since then, the content of concept of safety is much relevant to homeland security and personal safety against terrorism. The aftermath of the attacks also clearly demonstrates the spirit of the civilized world and the enormous capacity of this world to unite; to coordinate efforts among civilized countries, organizations, as well as among private businesses, community groups, and individual citizens in response to a crisis; and to make the sacrifices necessary to respond both to these new threats and the consequences they entail.

Our challenge is to build upon a renewed purpose in ways that create both short- and long-term benefits and allow us to sustain our efforts. As a result, we must find the best ways to sustain our efforts over a significant time period and leverage our finite resources in ways that will have the greatest effects. 
An effective framework to address these challenges will require a risk management approach to focus finite resources on areas of greatest need.

However, although the United States of America has the greatest number of high-technology tools, until now it does not have a comprehensive risk management approach [5] to help guiding federal programs for homeland security and apply their resources efficiently and to best effect. One of the main reasons why it is difficult to promote risk management in the new safety issue is that users have little confidence in the results of their risk analysis from incomplete data in a short time for a very complex system. In this paper, we will suggest an approach to study the information described in natural language for improving risk analysis.

This paper is organized as follows. In Section 2, we give a new definition of risk and a sample scene of risk. We outline of some methodologies for risk analysis and indicate their disadvantage in Section 3. We suggest an approach to infer risks with information described in natural language in Section 4. With fuzzy logic, in Section 5, we give a sample to verify that the suggested approach is more flexible and effective. We conclude the paper in Section 6.

\section{A New Definition of Risk}

Safety is the state of being certain that adverse effects will not be caused by some agent under defined conditions. The reciprocal of safety is risk.

There is no widely accepted definition of risk. Four items are found for risk in Webster Dictionary:

(1) Possibility of loss or injury;

(2) Someone or something that creates or suggests a hazard;

(3) a: The chance of loss or the perils to the subject matter of an insurance contract; also: the degree of probability of such loss.

$\mathrm{b}$ : A person or thing that is a specified hazard to an insurer $<$ a poor risk for insurance $>$.

c: An insurance hazard from a specified cause or source $<$ war risk $>$.

(4) The chance that an investment (as a stock or commodity) will lose value.

Obviously, the core of all definitions of risk is the same: risk exists when loss is possible and its financial impact is significant. This linguistic definition captures a property of risk that eludes definition in terms of mathematical formulas where risk had been defined as the probability per unit time of the occurrence of a unit cost burden [4].

But, what on earth is risk, anyway? Is it "loss"? "probability"? or "probability of loss"? No, they are not. They are at most features of risk. In fact, risk is a synthetic phenomenon that would be described in Definition 1.

Definition 1. Risk is a scene in the future associated with some adverse incident. 
Scene means something seen by a viewer; a view or prospect. Adverse is contrary to one's interests or welfare; harmful or unfavorable.

A scene must be described with a system consisting of time, site and objects. The association would be measured with a metric space (such as probability). And, incident would be scaled with a magnitude (such as loss).

It is interesting to notice that, the concept of risk in Chinese is represented in two Chinese characters: "feng" and "xian," the former means "wind", and the latter "danger."

The risk meaning described in Chinese leads to a sample scene: a stone is hooked over our head. When a wind comes, the stone would fall down on our head. If the stone is small, or the hook can firmly hold the stone, any wind doesn't matter for us with respect to the stone. Otherwise, we have to take some prevention measure. Meanwhile, one day, the stone may fall down without any wind if the hook is made of wood. In the scene, there are three objects: stone, wind and our head. The wind would be replace by another force. For example, moth eats the wooden hook.

\section{Outline of Some Methodologies for Risk Analysis}

Risk management is the process of evaluating and, if necessary, controlling sources of exposure and risk. The culture, processes and structures that are directed towards the effective management of potential opportunities and adverse effects.

Risk evaluation is the process used to determine risk management priorities by comparing the level of risk against predetermined standards, target risk levels or other criteria.

Risk analysis is a systematic use of available information to determine how often specified events may occur and the magnitude of their consequences.

Risk assessment is the overall process of risk analysis and risk evaluation.

Strictly speaking, risk management covers the hazard identification and risk analysis, but focuses on executing, monitoring and controlling. On the other hand, risk analysts often divide risk analysis into these components: risk assessment, risk management and risk communication.

According to Definition 1, we know that the risk is a scene. Therefore, we refer to risk management as an appropriate activation so that our environment will become more safe, and refer to risk analysis as a scientifically description of a risk system so that the risk management will become more effective.

There have been appearing a lot of risk analysis methodologies. Among them, the following 11 methodologies are more popular.

(1) Failure Modes, Effects and Criticality Analysis (FMECA);

(2) Failure Mode and Effect Analysis (FMEA);

(3) Fault Tree Analysis (FTA);

(4) Hazard and Operability Analysis (HAZOP);

(5) Cause Consequence Analysis (CCA);

(6) Management Oversight Risk Tree (MORT); 
(7) Safety Management Organization Review Technique (SMORT);

(8) Risk Analysis Bibliographies (RAB);

(9) Security/Survivability Systems Analysis (S/SSA);

(10) Cost-Effectiveness Analysis in Emergency Medicine, Computer (CEA);

(11) Cost Benefit Analysis (CAB)

Most of them involve the assumption that a risk system can be decomposed into several subsystems where the corresponding risks are known. For example, FTA is a deductive procedure for determining the various combinations of hardware and software failures, and human errors that could result in the occurrence of specified undesired events. Another example: one of basic steps in an FMEA is to enter the probability factor where a numerical weight should be assigned to each cause that indicates how likely that cause is (probability of the cause occurring).

In the cognitive viewpoint, there are four different types of societal risks [3]:

(T1) Real risk to an individual - eventually by future circumstances when they fully develop;

(T2) Statistical risk - determined by currently available data, typically measured actuarially for insurance premium and other purposes;

(T3) Predicted risk — predicted analytically from system models structured from historical studies;

(T4) Perceived risk — intuitively seen by individuals.

Therefore, the methodologies for risk analysis also can be specified four categorizations: (1) Investigating the current scene; (2) Statistically inferencing; (3) Modelling the future scene; (4) Evaluating with expert experiences.

Obviously, the core of risk analysis is not to combine the subsystems but to analyze the subsystems. The main disadvantage of these methodologies is that they depend on so much assumptions for subsystems, such as that we can find a statistical rule to show the risk phenomenon properly, or find some state equations to describe risk systems, or acquire expert experiences to understand the risk phenomenon. The real cases told us, it is difficult to verify if the assumptions are reasonable.

Beck 1] viewed the increasing focus on risk as the consciousness of a new stage of development in society. The return of uncertainty is the difference between an industrial society and a risk society. If it is barely possible to analyze risks in industrial societies with explicit assumptions, it will be impossible to analyze risks in post-modern societies with these assumptions. In many cases, only a few pieces of information in massive information are available; the environment is not clear and we are required to response quickly. We have to make a decision in an uncertain environment with fuzzy information as soon as possible. It is impossible to accurately analyze risks in complex systems. In the future, we would have to analyze risks by computing with words [7], or with images, in which we would process fuzzy information more efficiently. Fuzzy set theory is created to computing with words. 


\section{Inferring Risks with Information Described in Natural Language}

Computation with information described in natural language, or NLcomputation for short, is a problem of intrinsic importance because much of human knowledge is described in natural language 8

Basically, NL-Computation is a system of computation in which the objects of computation are words and propositions drawn from a natural language. NLComputation is reduced to computation with generalized constraints, that is, to generalized-constraint-based computation. NL-Computation is based on fuzzy logic. NL-Computation is closely related to Computing with Words (CW).

Here is an example of NL-Computation in risk analysis. A middle stone is hooked over our head with a wooden hook. If a storm comes, the stone would fall down on our head. It is called a stone-hook-wind system (SHW-system). Our task is to analyze what risk, with high possibility, we have to bear if we don't take any prevention measure?

Let $E$ be risk in values: yes (the stone will fall down) or no, $X$ be the features of the hook (strength and fastness), and $Y$ be the features of the storm (force and frequency). $X, Y$ are imprecisely described in natural language. Particularly, $Y$ is a fuzzy random vector. The risk analysis is to calculate:

$$
\operatorname{Prob}(E=y e s) \text { with high possibility. }
$$

In general, we are used to calculate $\operatorname{Prob}(E=y e s)$ by using observations based on some distribution assumptions or non-parametric method. However, now, we haven't any observation, but only information described in natural language about stone, hook and wind. It is impossible to directly calculate $\operatorname{Prob}(E=y e s)$ by using any traditional method.

The approach to NL-computation centers on what is referred to as generalizedconstraint-based computation. A generalized constraint is expressed as " $X$ is $r$ $A$," where $X$ is the constrained variable, $A$ is a constraining relation and $r$ is an indexical variable which defines the way in which $A$ constrains $X$.

NL-computation cannot be dealt with through the use of machinery of natural language processing. The problem is semantic imprecision of natural languages. More specifically, a natural language is basically a system for describing perceptions. Perceptions are intrinsically imprecise, reflecting the bounded ability of sensory organs, and ultimately the brain, to resolve detail and store information. Semantic imprecision of natural languages is a concomitant of imprecision of perceptions.

To a SHW-system, there is no loss of generality in assuming that " $X$ is $r_{1} A$ " and " $Y$ is $r_{2} B$ ", where

$$
\begin{gathered}
X=\left\{X_{1}, X_{2}\right\}=\{\text { strength }, \text { fastness }\} \\
Y=\left\{Y_{1}, Y_{2}\right\}=\{\text { force }, \text { frequency }\}
\end{gathered}
$$




$$
\begin{gathered}
r_{1}=\left\{r_{11}, r_{12}\right\}=\{\text { blank, blank }\}, \\
r_{2}=\left\{r_{21}, r_{22}\right\}=\{p, p\},
\end{gathered}
$$

where blank means the possibilistic constraint and $p$ means the probabilistic constraint. Then,

$$
\begin{aligned}
& \operatorname{Prob}(E=y e s) \text { with high possibility } \\
& =\operatorname{Prob}(B \mid E=\text { yes with high possibility determined by } A) .
\end{aligned}
$$

\section{A Calculation Case}

The information for our case is that a middle stone is hooked over our head with a strong wooden hook almost fast. If the hook doesn't hold the stone absolutely fast, when a wind comes, the stone would fall down. The winds more than 40 $\mathrm{km} / \mathrm{h}$ have been frequently observed in this region.

What risk do we face?

Let $E$ be risk in values: 1 or 0 (down or not down). Let $X$ be the fastness of that the hook holds the stone, with universe:

$$
U=\left\{u_{1}, u_{2}, \cdots, u_{11}\right\}=\{0,0.1, \cdots, 1\},
$$

$Y$ be the intensity $(\mathrm{km} / \mathrm{h})$ of a wind, with universe:

$$
V=\left\{v_{1}, v_{2}, \cdots, v_{12}\right\}=\{0,10, \cdots, 110\} .
$$

From the information about the SHW-system, specifically described in natural language, we obtain:

$$
X \text { is } A
$$

where

$$
A \triangleq \text { almost fast }
$$

with membership function

$$
\mu_{A}(u)=\frac{0.3}{0.6}+\frac{0.6}{0.7}+\frac{1}{0.8}+\frac{0.6}{0.9}+\frac{0.3}{1} .
$$

Suppose that, from information "If the hook doesn't hold the stone absolutely fast, when a wind comes, the stone would fall down," we obtain a falling table determined by $u$ and $v$, shown in Table 1 , where 1 means that the stone will fall down, and 0 means the hook can hold the stone.

We suppose that, the information "The winds more than $40 \mathrm{~km} / \mathrm{h}$ have been frequently observed in this region" can be interpreted as that the average intensity of the wind is $40 \mathrm{~km} / \mathrm{h}$. Because the number of weak winds is much more than the number of strong winds, we suppose the probability distribution of the intensity is exponential. 
Table 1. Falling table determined by the fastness and the intensity

\begin{tabular}{|c|llllllllllll|}
\hline & \multicolumn{11}{c|}{ Intensity $(\mathrm{km} / \mathrm{h})$} \\
Fastness & 0 & 10 & 20 & 30 & 40 & 50 & 60 & 70 & 80 & 90 & 100 & 110 \\
\hline 0 & 1 & 1 & 1 & 1 & 1 & 1 & 1 & 1 & 1 & 1 & 1 & 1 \\
0.1 & 0 & 0.9 & 1 & 1 & 1 & 1 & 1 & 1 & 1 & 1 & 1 & 1 \\
0.2 & 0 & 0.6 & 0.9 & 1 & 1 & 1 & 1 & 1 & 1 & 1 & 1 & 1 \\
0.3 & 0 & 0.3 & 0.6 & 0.9 & 1 & 1 & 1 & 1 & 1 & 1 & 1 & 1 \\
0.4 & 0 & 0 & 0.3 & 0.6 & 0.9 & 1 & 1 & 1 & 1 & 1 & 1 & 1 \\
0.5 & 0 & 0 & 0 & 0.3 & 0.6 & 0.9 & 1 & 1 & 1 & 1 & 1 & 1 \\
0.6 & 0 & 0 & 0 & 0 & 0.3 & 0.6 & 0.9 & 1 & 1 & 1 & 1 & 1 \\
0.7 & 0 & 0 & 0 & 0 & 0 & 0.3 & 0.6 & 0.9 & 1 & 1 & 1 & 1 \\
0.8 & 0 & 0 & 0 & 0 & 0 & 0 & 0.3 & 0.6 & 0.9 & 1 & 1 & 1 \\
0.9 & 0 & 0 & 0 & 0 & 0 & 0 & 0 & 0.3 & 0.6 & 0.9 & 1 & 1 \\
1 & 0 & 0 & 0 & 0 & 0 & 0 & 0 & 0 & 0 & 0 & 0 & 0 \\
\hline
\end{tabular}

Then, we obtain a probability distribution of the intensity of a wind:

$$
p(v)=0.025 e^{-0.025 v} .
$$

Table 1 can be regarded as a fuzzy relation matrix between fastness and intensity, constrained by falling phenomena. From input "almost fast", with the max-min composition [2, we obtain an output B, called strong wind, that makes the stone falls, with membership function:

$$
\mu_{B}(v)=\frac{0.3}{40}+\frac{0.3}{50}+\frac{0.3}{60}+\frac{0.6}{70}+\frac{0.9}{80}+\frac{1}{90}+\frac{1}{100}+\frac{1}{110} .
$$

The probability of the fuzzy event is [6]:

$$
\begin{aligned}
P(B) \approx & \int_{0}^{110} \mu_{B}(v) p(v) d v \\
\approx & 0.025\left(0.3 \int_{35}^{65} e^{-0.025 v} d v+0.6 \int_{65}^{75} e^{-0.025 v} d v+0.9 \int_{75}^{85} e^{-0.025 v} d v\right. \\
& \left.+\int_{85}^{110} e^{-0.025 v} d v\right) \\
= & 0.178154 .
\end{aligned}
$$

Therefore, we know that, the risk we face is

$$
\begin{aligned}
& \operatorname{Prob}(E=y e s) \text { with high possibility } \\
& =\operatorname{Prob}(B \mid E=1 \text { with high possibility determined by } A) \\
& =\operatorname{Prob}(B) \\
& =0.178154
\end{aligned}
$$


i.e., the probability of that the stone falls is 0.178154 with high possibility. From the given information described in natural language, it is impossible to accurately calculate the probability of the falling of the stone. Here, the probability value 0.178154 is induced by using fuzzy logic. Because the inference is comparatively reliable, the value 0.178154 can be regarded one near real probability with high possibility.

\section{Conclusion and Discussion}

Risk is a scene in the future associated with some adverse incident. This linguistic definition can capture all properties of risk that eludes definition in terms of mathematical formulas.

It is possible to analyze risk with information described in natural language. The fuzzy logic can be used to quantify the information and infer the risk where computation with information described in natural language is reduced to computation with generalized constraints, that is, to generalized-constraint-based computation.

The basic condition to compute risk with information described in natural language is that we can understand the semantic in the information and change it into constraints. With the same information described in natural language, different experts would infer different risks due to different backgrounds. Therefore, any risk inferred from the natural language is soft. To limit the soft degree, it is necessary to form a set of kernel terms used to describe risk so that different experts have almost same understanding for each term in the set.

\section{References}

1. Beck, U.: Risk Society, Towards a New Modernity. Sage Publications, London (1992)

2. Huang, C.F., Shi, Y.: Towards Efficient Fuzzy Information Processing - Using the Principle of Information Diffusion. Physica-Verlag, Heidelberg (2002)

3. Sage, A.P., White, E.B.: Methodologies for Risk and Hazard Assessment: a Survey and Status Report. IEEE Trans. Systems, Man, and Cybernetics, SMC-10(8) (1980) 425-446

4. Starr, C., Whipple, C.: Risks of Risk Decisions. Science, 208(4448) (1980) 1114-1119

5. United States General Accounting Office: Homeland Security: Challenges in Creating an Effective Acquisition Organization. GAO-06-1012T, Washington D.C., 2006

6. Zadeh, L.A.: Fuzzy Probabilities. Information Processing Management, 20(3) (1984) 363-372

7. Zadeh, L.A.: Fuzzy Logic $=$ Computing with Words. IEEE Trans. Fuzzy Systems, 4(2) (1996) 103-111

8. Zadeh, L.A.: Computation with Information Described in Natural Language - The Concept of Generalized-Constraint-Based Computation. In: Ruan, D., D'hondt, P., Fantoni, P.F., De Cock, M., Nachtegael, M., Kerre, E.E. (eds.): Applied Artificial Intelligence. Proceedings of the 7th International FLINS Conference (Genova, Italy, August 29-31, 2006). World Scientific, Singapore (2006) 3-4 